\title{
NIR LINE OBSERVATIONS OF STARBURST GALAXIES
}

\author{
H. SUGAI \\ Dept. of Astron., Kyoto Univ., Japan \\ M.A. MALKAN \\ Dept. of Astron., Univ. of California at Los Angeles, USA \\ M.J. WARD \\ Dept. of Physics and Astron., Univ. of Leicester, UK \\ R.I. DAVIES \\ Dept. of Physics, Nuclear Physics Laboratory, UK \\ AND
}

I.S. MCLEAN

Dept. of Astron., Univ. of California at Los Angeles, USA

We have obtained images of the $\mathrm{H}_{2}$ and $\mathrm{Br} \gamma$ emission lines in the galaxy interacting system NGC 3690 + IC 694 . We have also obtained simultaneous $\mathrm{H}$ - and $\mathrm{K}$-band spectra for three of its $2 \mu \mathrm{m}$ continuum peaks. The most detectable line emission is concentrated at the continuum peaks. Therefore, the emission lines as well as stellar absorption lines can be used as tracers of the activity in the nuclei themselves. From the strong $\mathrm{Br} \gamma$ and marginal detection of $\operatorname{Br} 10$ at the nucleus of IC 694 , we derive a large extinction for the fully ionized gas in this nucleus. If we adopt this extinction also for the [Fe II] $1.64 \mu \mathrm{m}$ emission, the extinction-corrected [Fe II] $1.64 \mu \mathrm{m} / \mathrm{Br} \gamma$ ratio will lie at the higher end of starburst galaxies, and is typical for AGNs or AGN/starburst composites. This might imply that many SNRs are involved in the starburst at this nucleus, unless it includes an AGN. All of our results for Component $\mathrm{C}$, including very little $\mathrm{CO}$ absorption in the $\mathrm{K}$ band, a large $E W(\mathrm{Br} \gamma)$, a small $\mathrm{H}_{2} / \mathrm{Br} \gamma$ ratio, the effective temperature $\left(T_{\text {eff }} \simeq 40,000 \mathrm{~K}\right)$ derived from $\mathrm{HeI} 1.70 \mu \mathrm{m} / \mathrm{Br} 10$ and $\mathrm{HeI} 2.06 \mu \mathrm{m} / \mathrm{Br} \gamma$, are consistent with a very young starburst.

We have also obtained an $\mathrm{H}_{2}$ image of another galaxy interacting system Mrk 551, as well as its spectra. In contrast to NGC 3690 + IC 694, we have found that the $\mathrm{H}_{2}$ emission peak is offset from the most luminous continuum peak by several hundred parsecs. 